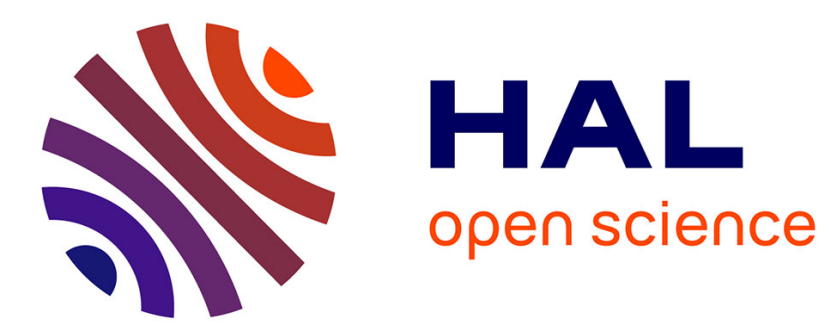

\title{
Attitude, Linear Velocity and Depth Estimation of a Camera observing a planar target using continuous homography and inertial data
}

\author{
Minh-Duc Hua, Ninad Manerikar, Tarek Hamel, Claude Samson
}

\section{To cite this version:}

Minh-Duc Hua, Ninad Manerikar, Tarek Hamel, Claude Samson. Attitude, Linear Velocity and Depth Estimation of a Camera observing a planar target using continuous homography and inertial data. IEEE International Conference on Robotics and Automation (ICRA), May 2018, Brisbane, Australia. hal-01878711

\author{
HAL Id: hal-01878711 \\ https://hal.science/hal-01878711
}

Submitted on 21 Sep 2018

HAL is a multi-disciplinary open access archive for the deposit and dissemination of scientific research documents, whether they are published or not. The documents may come from teaching and research institutions in France or abroad, or from public or private research centers.
L'archive ouverte pluridisciplinaire HAL, est destinée au dépôt et à la diffusion de documents scientifiques de niveau recherche, publiés ou non, émanant des établissements d'enseignement et de recherche français ou étrangers, des laboratoires publics ou privés. 


\title{
Attitude, Linear Velocity and Depth Estimation of a Camera observing a planar target using continuous homography and inertial data
}

\author{
Minh-Duc Hua, Ninad Manerikar, Tarek Hamel, Claude Samson
}

\begin{abstract}
This paper revisits the problem of estimating the attitude, linear velocity and depth of an IMU-Camera with respect to a planar target. The considered solution relies on the measurement of the optical flow (extracted from the continuous homography) complemented with gyrometer and accelerometer measurements. The proposed deterministic observer is accompanied with an observability analysis that points out camera's motion excitation conditions whose satisfaction grants stability of the observer and convergence of the estimation errors to zero. The performance of the observer is illustrated by performing experiments on a test-bed IMU-Camera system.
\end{abstract}

\section{INTRODUCTION}

The recent proliferation of mini Unmanned Aerial Vehicles (UAVs), the emergence of modern embedded computing, and the availability of low-cost MEMS sensor systems have opened a vast range of new civil applications such as traffic congestion monitoring, environmental sensing, infrastructure inspection, real estate photography, and hazard detection and surveillance. In practice, most of these applications require the UAVs to fly in close proximity to the physical environment with GPS signals that are sometimes unavailable or unreliable. A number of research groups work actively on associated technical issues. Significant advances have also been obtained in the last few years in the domain of controlling aerial robotic vehicles. We may cite, for instance, quadrotor landing on inclined surfaces [26], quadrotor manoeuvring with a cable-suspended payload [25], inverted pendulum balancing and catching [3]. However, in these examples the local environment is not taken into account. The control algorithms rely on full state measurements obtained from an external 3D localization system, which limits their applicability to a suitably equipped experimental flight area. Documented results for aerial robotic vehicles in a GPS-denied environment using onboard exteroceptive sensors, such as laser range finders or vision, involve far less aggressive manoeuvres [7], [24]. A central issue is thus the estimation of the vehicle's state relatively to its environment via the use of sensors that do not measure this state directly. It is only in the last five years or so that researchers have begun to tackle this issue by focusing primarily on the problem of attitude estimation when the vehicle undergoes sustained accelerations. This led to the development of velocity-aided attitude observers that fuse Inertial Measurement Unit (IMU) readings with the linear velocity measurements [2], [6], [10],

M.-D. Hua, N. Manerikar, T. Hamel are with I3S, Université Côte d'Azur, CNRS, Sophia Antipolis, France, hua(manerika,hamel)@i3s.unice.fr.

C. Samson is with INRIA and I3S UCA-CNRS, Sophia Antipolis, France, claude.samson@inria.fr,csamson@i3s.unice.fr.
[12], [15], [18], [22]. The lack of an onboard linear velocity sensor for mini UAVs led other researchers to exploit the vehicle's dynamics equations instead [1], [11], [16], [19]. Previously mentioned examples exploit proprioceptive sensor modalities whereas typical tasks involve interaction of the robotic vehicle with the environment. Combining a vision system with an IMU recently led to the development of integrated observers that exploit optical flow measurements and IMU readings to estimate the camera's attitude, linear velocity, and its distance to a planar target [4], [5], [27]. The standard approach consists in applying extended Kalman filters and showing experimentally the practical convergence of the estimation errors [27]. However, observability and convergence analyses are either missing or incomplete in these studies. An alternative solution is to use deterministic observer design techniques, alike those reported in [4], [5] except that the algorithms proposed in theses references rely on the strong assumption of an IMU providing measurements of the vehicle's linear acceleration expressed in the bodyfixed frame ${ }^{1}$.

In the present paper, the problem of attitude, linear velocity and depth estimation is revisited by also adopting a deterministic observer point of view, but without relying on the assumption used in [4], [5]. The proposed observer is adapted from the deterministic Riccati observer design framework derived in [8]. In contrast with most existing works on the same topic [27], the structural question of observability, on which the exponential stability and convergence of the observer rely, is here addressed with the derivation of an explicit (and simple) observability condition based on the persistence of excitation granted by the camera-IMU linear velocity. Several practical algorithmic and implementation issues are also discussed.

The paper is organized as follows. Notation, system equations, and the measurements involved in the observer design are specified in Section II. In the same section a few basic definitions and conditions about uniform observability are recalled, together with elements of the deterministic Riccati observer design framework proposed in [8]. In Section III the proposed observer is specified, and an analysis of associated observability conditions is carried out. Section IV describes practical implementation aspects of this observer. Experimental results illustrating its performance is reported in Section V. A concluding section follows.

\footnotetext{
${ }^{1}$ In fact, accelerometers only provide measurements of the so-called specific acceleration.
} 


\section{Preliminary MATERIAL}

\section{A. Notation}

- $\{\mathcal{I}\}$ denotes an inertial frame attached to the earth, typically chosen as the north-east-down frame, and $\{\mathcal{B}\}$ is a body-fixed frame attached to the vehicle.

- The vehicle's attitude is represented by a rotation matrix $R \in \mathrm{SO}(3)$ of the frame $\{\mathcal{B}\}$ relative to $\{\mathcal{I}\}$.

- $v \in \mathbb{R}^{3}$ and $\omega \in \mathbb{R}^{3}$ are the vectors of coordinates of the vehicle's linear and angular velocities expressed in $\{\mathcal{B}\}$.

- $\left\{e_{1}, e_{2}, e_{3}\right\}$ denotes the canonical basis of $\mathbb{R}^{3}$ and $[\cdot]_{\times}$ denotes the skew-symmetric matrix associated with the cross product, i.e., $[u]_{\times} v=u \times v, \forall u, v \in \mathbb{R}^{3}$. The identity matrix of $\mathbb{R}^{n \times n}$ is denoted as $I_{n}$ and $\pi_{x} \triangleq I_{3}-x x^{\top}, \forall x \in S^{2}$ (the unit 2-sphere), is the projection operator onto the plane orthogonal to $x$. Note that $\pi_{x}=-[x]_{\times}^{2}, \forall x \in S^{2}$.

\section{B. System equations and measurements}

The vehicle's attitude satisfies the differential equation

$$
\dot{R}=R[\omega]_{\times}
$$

It is assumed that the vehicle is equipped with an IMU comprising a 3-axis gyrometer that measures the angular velocity $\omega \in \mathbb{R}^{3}$ and a 3 -axis accelerometer that measures the so-called specific acceleration $a_{\mathcal{B}} \in \mathbb{R}^{3}$, expressed in $\{\mathcal{B}\}$. Using the flat non-rotating Earth assumption, one has [2]

$$
\dot{v}=-[\omega]_{\times} v+a_{\mathcal{B}}+g R^{\top} e_{3}
$$

where $g$ is the gravity constant. A 3-axis magnetometer is also assumed to be available to measure of the normalized Earth's magnetic field vector expressed in $\{\mathcal{B}\}$. Let $m_{\mathcal{I}} \in$ $S^{2}$ denote the known normalized Earth's magnetic field vector expressed in $\{\mathcal{I}\}$. The vectors $m_{\mathcal{I}}$ and $e_{3}$ are usually assumed to be non-collinear so that $R$ can be estimated from the observation (measurements) in the body-fixed frame of the gravity vector and of the Earth's magnetic field vector. The magnetometer thus measures $m_{\mathcal{B}}=R^{\top} m_{\mathcal{I}}$.

We further assume that the vehicle is equipped with a monocular camera that observes a planar scene so that we can obtain an estimation of the so-called continuous homography [17, Ch.5]:

$$
H_{c}=[\omega]_{\times}+\frac{v}{d} \eta^{\top}
$$

where $\eta \in S^{2}$ is the unit normal vector to the plane and $d>$ 0 is the distance from the camera to the plane. By extracting the term $[\omega]_{\times}$from $H_{c}$ using gyrometer measurements, one obtains $U \triangleq \frac{v}{d} \eta^{\top}=H_{c}-[\omega]_{\times}$and, thus, $U U^{\top}=\frac{v}{d} \frac{v^{\top}}{d}$. Then, by simple decomposition of $U U^{\top}$ one obtains $\frac{v}{d}$. One also has

$$
\phi \triangleq \operatorname{trace}(U)=\operatorname{trace}\left(\frac{v^{\top} \eta}{d}\right)=-\frac{\dot{d}}{d}
$$

Defining $s \triangleq \frac{1}{d}$ one deduces

$$
\dot{s}=-\frac{\dot{d}}{d^{2}}=\phi s
$$

In summary, the observer design for $R, v$ and $s$ (i.e. $d$ ) will be based on Eqs. (1)-(3) and the measured quantities $\frac{v}{d}, \phi, \omega, a_{\mathcal{B}}$ and $m_{\mathcal{B}}$. We will also show thereafter that the magnetometer measurements $m_{\mathcal{B}}$ are not required if we only need to estimate the gravity direction (i.e. $R^{\top} e_{3}$ ) instead of the whole attitude $R$.

\section{Recalls of uniform observability}

Consider a linear time-varying (LTV) system

$$
\begin{cases}\dot{x} & =A(t) x+B(t) u \\ y & =C(t) x\end{cases}
$$

with $x \in \mathbb{R}^{n}$ the system state vector, $u \in \mathbb{R}^{s}$ the system input vector, and $y \in \mathbb{R}^{m}$ the system output vector.

Definition 1 (uniform observability) System (4) is uniformly observable if there exist $\delta, \mu>0$ such that (s.t.) $\forall t \geq 0$

$$
W(t, t+\delta) \triangleq \frac{1}{\delta} \int_{t}^{t+\delta} \Phi^{\top}(t, \tau) C^{\top}(\tau) C(\tau) \Phi(t, \tau) d \tau
$$

with $\Phi(t, \tau)$ the transition matrix associated with $A(t)$, i.e. such that $\frac{d}{d t} \Phi(t, \tau)=A(t) \Phi(t, \tau)$ with $\Phi(t, t)=I_{n}$.

$W(t, t+\delta)$ is called the observability Gramian of System (4). One also says that the pair $(A(t), C(t))$ is uniformly observable when (5) is satisfied.

Lemma 1 (see [23]) If there exists a matrix-valued function $M(\cdot)$ of dimension $(p \times n)(p \geq 1)$ composed of row vectors of $N_{0}=C, N_{k}=N_{k-1} A+\dot{N}_{k-1}, k=1, \cdots$ such that for some positive numbers $\bar{\delta}, \bar{\mu}$ and $\forall t \geq 0$

$$
\frac{1}{\bar{\delta}} \int_{t}^{t+\bar{\delta}} M^{\top}(\tau) M(\tau) d \tau \geq \bar{\mu} I_{n}
$$

then the observability Gramian of System (4) satisfies condition (5).

\section{Recalls of a Riccati observer design framework}

The design of the proposed observer in this paper is adapted from the deterministic observer design framework developed in [8]. Consider the nonlinear system

$$
\begin{cases}\dot{x} & =A\left(x_{1}, t\right) x+u \\ y & =C(x, t) x\end{cases}
$$

with $x=\left[x_{1}^{\top}, x_{2}^{\top}\right]^{\top}, x_{1} \in \mathcal{B}_{r}^{n_{1}}$ (the closed ball in $\mathbb{R}^{n_{1}}$ of radius $r), x_{2} \in \mathbb{R}^{n_{2}}, y \in \mathbb{R}^{m}, A\left(x_{1}, t\right)$ a continuous matrixvalued function uniformly bounded with respect to (w.r.t.) $t$ and uniformly continuous w.r.t. $x_{1}$ of the form

$$
A\left(x_{1}, t\right)=\left[\begin{array}{cc}
A_{1,1}(t) & 0_{n_{1} \times n_{2}} \\
A_{2,1}\left(x_{1}, t\right) & A_{2,2}(t)
\end{array}\right]
$$

and $C(x, t) \in \mathbb{R}^{m \times\left(n_{1}+n_{2}\right)}$ a continuous matrix-valued function uniformly bounded w.r.t. $t$ and uniformly continuous w.r.t. $x$. Apply the input

$$
u=-P C^{\top} Q y
$$

with $P \in \mathbb{R}^{\left(n_{1}+n_{2}\right) \times\left(n_{1}+n_{2}\right)}$ a symmetric positive definite matrix solution to the following continuous Riccati equation (CRE):

$$
\dot{P}=A P+P A^{\top}-P C^{\top} Q(t) C P+V(t)
$$

with $P(0) \in \mathbb{R}^{\left(n_{1}+n_{2}\right) \times\left(n_{1}+n_{2}\right)}$ a symmetric positive definite matrix, $Q(t) \in \mathbb{R}^{m \times m}$ bounded continuous symmetric positive semidefinite, and $V(t) \in \mathbb{R}^{\left(n_{1}+n_{2}\right) \times\left(n_{1}+n_{2}\right)}$ bounded continuous symmetric positive definite. Then, from Theorem 3.1 and Corollary 3.2 in [8], $x=0$ is locally exponentially 
stable when $Q(t)$ and $V(t)$ are both larger than some positive matrix and the pair $\left(A^{\star}(t), C^{\star}(t)\right)$, with $A^{\star}(t) \triangleq A(0, t)$, $C^{\star}(t) \triangleq C(0, t)$, is uniformly observable. It is proved in [8, Theorem 3.1] that this local exponential stability result is also valid for first order approximations.

\section{RICCATI OBSERVER DESIGN}

\section{A. Observer derivation}

Let $\hat{R} \in \mathrm{SO}(3), \hat{v} \in \mathbb{R}^{3}, \hat{s} \in \mathbb{R}$ denote the estimates of $R, v, s$, respectively. The proposed observer is given by

$$
\left\{\begin{array}{l}
\dot{\hat{R}}=\hat{R}[\omega]_{\times}-\left[\sigma_{R}\right]_{\times} \hat{R} \\
\dot{\hat{v}}=-[\omega]_{\times} \hat{v}+a_{\mathcal{B}}+g \hat{R}^{\top} e_{3}-\sigma_{v} \\
\dot{\hat{s}}=\phi \hat{s}-\sigma_{s}
\end{array}\right.
$$

where $\sigma_{R}, \sigma_{v} \in \mathbb{R}^{3}, \sigma_{s} \in \mathbb{R}$ are innovation terms to be designed thereafter. Defining the observer errors

$$
\tilde{R} \triangleq R \hat{R}^{\top}, \tilde{v} \triangleq v-\hat{v}, \tilde{s} \triangleq s-\hat{s}
$$

then the observer's objective can be stated as the exponential stability of $(\tilde{R}, \tilde{v}, \tilde{s})=\left(I_{3}, 0,0\right)$ (or of $\left(\tilde{R} e_{3}, \tilde{v}, \tilde{s}\right)=$ $\left(e_{3}, 0,0\right)$ when the estimation of the gravity direction is concerned instead of the whole attitude estimation).

From (1), (2), (10), one verifies that the dynamics of $(\tilde{R}, \tilde{v}, \tilde{s})$ satisfy

$$
\left\{\begin{array}{l}
\dot{\tilde{R}}=\tilde{R}\left[\sigma_{R}\right]_{\times} \\
\dot{\tilde{v}}=-[\omega]_{\times} \tilde{v}+g \hat{R}^{\top}\left(\tilde{R}^{\top}-I_{3}\right) e_{3}+\sigma_{v} \\
\dot{\tilde{s}}=\phi \tilde{s}+\sigma_{s}
\end{array}\right.
$$

We will work out next first order approximations of the error system (11) complemented with first order approximations of the measurement equations. The application to these approximations of the Riccati observer design framework reported in [8] (see Section II-D) will then provide us with the equations of the innovation terms of the proposed observer.

For this application the following technical (but nonrestrictive) assumption is made.

Assumption $1 v(t), \dot{v}(t), \omega(t)$ and $\phi$ are bounded in norm by some positive numbers $v_{\max }, \dot{v}_{\max }, \omega_{\max }$ and $\phi_{\max }$, respectively. The distance $d$ is lower- and upper-bounded by some positive numbers $d_{\min }$ and $d_{\max }$, respectively.

First order approximations of the attitude error equations are derived using a (local) minimal parametrization of the rotation group $\mathrm{SO}(3)$. The parametrization here chosen is the vector part $\tilde{q}_{v}$ of the Rodrigues unit quaternion $\tilde{q}=\left(\tilde{q}_{0}, \tilde{q}_{v}\right)$ associated with $\tilde{R}$. Rodrigues formula relating $\tilde{q}$ to $\tilde{R}$ is

$$
\tilde{R}=I_{3}+2\left[\tilde{q}_{v}\right]_{\times}\left(\tilde{q}_{0} I_{3}+\left[\tilde{q}_{v}\right]_{\times}\right)
$$

From this relation, one deduces

$$
\tilde{R}=I_{3}+[\tilde{\lambda}]_{\times}+O\left(|\tilde{\lambda}|^{2}\right), \quad \text { with } \tilde{\lambda} \triangleq 2 \operatorname{sign}\left(\tilde{q}_{0}\right) \tilde{q}_{v}
$$

Then, in view of the dynamics of $\tilde{R}$ in (11) one verifies (see also [8]) that the derivative of $\tilde{\lambda}$ is given by

$$
\dot{\tilde{\lambda}}=\sigma_{R}+O\left(|\tilde{\lambda}|\left|\sigma_{R}\right|\right)
$$

As for the dynamics of $\tilde{v}$ one obtains

$$
\begin{aligned}
\dot{\tilde{v}} & =-[\omega]_{\times} \tilde{v}+g \hat{R}^{\top}\left[e_{3}\right]_{\times} \tilde{\lambda}+\sigma_{v}+O\left(|\tilde{\lambda}|^{2}\right) \\
& =-[\omega]_{\times} \tilde{v}+g \hat{R}^{\top} e_{2} \tilde{\lambda}_{1}-g \hat{R}^{\top} e_{1} \tilde{\lambda}_{2}+\sigma_{v}
\end{aligned}
$$

with $\tilde{\lambda}_{1}, \tilde{\lambda}_{2}$ the first and second components of $\tilde{\lambda}$.

Concerning the measurement of $\frac{v}{d}$ one has

$$
\begin{aligned}
\frac{v}{d}-\hat{v} \hat{s} & =(\tilde{v}+\hat{v})(\tilde{s}+\hat{s})-\hat{v} \hat{s} \\
& =\left(\hat{s} I_{3}\right) \tilde{v}+\hat{v} \tilde{s}+O(|\tilde{v} \| \tilde{s}|)
\end{aligned}
$$

By setting the system output vector equal to

$$
y=\frac{v}{d}-\hat{v} \hat{s}
$$

one obtains LTV first order approximations in the form (7) with

$$
\left\{\begin{array}{l}
x=\left[\begin{array}{c}
\tilde{\lambda}_{1} \\
\tilde{\lambda}_{2} \\
\tilde{s} \\
\tilde{v}
\end{array}\right], x_{1}=\left[\begin{array}{c}
\tilde{\lambda}_{1} \\
\tilde{\lambda}_{2}
\end{array}\right], x_{2}=\left[\begin{array}{c}
\tilde{s} \\
\tilde{v}
\end{array}\right], u=\left[\begin{array}{c}
\sigma_{R, 1} \\
\sigma_{R, 2} \\
\sigma_{s} \\
\sigma_{v}
\end{array}\right] \\
A=\left[\begin{array}{cccc}
0 & 0 & 0 & 0_{1 \times 3} \\
0 & 0 & 0 & 0_{1 \times 3} \\
0 & 0 & \phi & 0_{1 \times 3} \\
g \hat{R}^{\top} e_{2} & -g \hat{R}^{\top} e_{1} & 0_{3 \times 1} & -[\omega]_{\times}
\end{array}\right] \in \mathbb{R}^{6 \times 6} \\
C=\left[\begin{array}{llll}
0_{3 \times 1} & 0_{3 \times 1} & \hat{v} & \hat{s} I_{3}
\end{array}\right] \in \mathbb{R}^{3 \times 6}
\end{array}\right.
$$

with $\sigma_{R, 1}, \sigma_{R, 2}$ the first and second components of $\sigma_{R}$. From there the proposed observer is given by (10) with $\sigma_{R, 1}, \sigma_{R, 2}$ and $\sigma_{v}$ determined from the input $u$ calculated according to (8) and (9).

As for the innovation component $\sigma_{R, 3}$, it can be independently designed for estimating the remaining degree of freedom of the attitude (i.e. yaw). This task is postponed and addressed thereafter. For instance, without loss of generality $\sigma_{R, 3}$ is assumed to be bounded for all time.

\section{B. Observability analysis}

According to [8, Corollary 3.2], good conditioning of the solutions $P(t)$ to the CREs and exponential stability of the proposed observer rely on the uniform observability of the pair $\left(A^{\star}(t), C^{\star}(t)\right)$ obtained by setting $x=0$ in the expressions of the matrices $A$ and $C$ given by (13). One verifies that

$$
\left\{\begin{aligned}
A^{\star} & =\left[\begin{array}{cccc}
0 & 0 & 0 & 0_{1 \times 3} \\
0 & 0 & 0 & 0_{1 \times 3} \\
0 & 0 & \phi & 0_{1 \times 3} \\
g \hat{R}^{\star \top} e_{2} & -g \hat{R}^{\star \top} e_{1} & 0_{3 \times 1} & -[\omega]_{\times}
\end{array}\right] \\
C^{\star} & =\left[\begin{array}{llll}
0_{3 \times 1} & 0_{3 \times 1} & v & s I_{3}
\end{array}\right]
\end{aligned}\right.
$$

with $\hat{R}^{\star} \in \mathrm{SO}(3)$ satisfying

$$
\hat{R}^{\star \top} e_{3}=R^{\top} e_{3}, \quad \dot{\hat{R}}^{\star}=\hat{R}^{\star}[\omega]_{\times}-\sigma_{R, 3}\left[e_{3}\right]_{\times} \hat{R}^{\star}
$$

Note that here $\hat{R}^{\star}$ is not equal to $R$ since $\tilde{\lambda}_{3}$ is not equal to zero when setting $x=0$.

From Lemma 1 the pair $\left(A^{\star}, C^{\star}\right)$ is uniformly observable if

$$
\exists \delta, \mu>0 \text { s.t. } \frac{1}{\delta} \int_{t}^{t+\delta} M^{\top}(\tau) M(\tau) d \tau \geq \mu I_{6}, \forall t>0
$$


with $M \triangleq\left[\begin{array}{c}C^{\star} \\ C^{\star} A^{\star}+\dot{C}^{\star}\end{array}\right]$. The next step consists in specifying a more explicit condition guaranteeing the satisfaction of (15).

Proposition 1 Assume that the following "persistent excitation" condition is satisfied: $\exists \delta, \rho>0$ and any index $i \in\{1,2,3\}$ such that

$$
\frac{1}{\delta} \int_{t}^{t+\delta}\left[\begin{array}{cc}
v_{i}(\tau)^{2} & s(\tau) v_{i}(\tau) \\
s(\tau) v_{i}(\tau) & s(\tau)^{2}
\end{array}\right] d \tau \geq \rho I_{2}, \forall t \geq 0
$$

Assume also that Assumption 1 holds. Then, condition (15) is satisfied. Consequently, the pair $\left(A^{\star}, C^{\star}\right)$ given by (14) is uniformly observable and the equilibrium $\left(\hat{R}^{\top} e_{3}, \hat{v}, \hat{s}\right)=$ $\left(R^{\top} e_{3}, v, s\right)$ of the proposed Riccati sub-observer is locally exponentially stable.

Proof: Using (16) and the boundedness of $v$ and $s$ according to Assumption 1, it is straightforward to show that there exists some positive number $\bar{\rho}>0$ such that

$$
\frac{1}{\delta} \int_{t}^{t+\delta}\left[\begin{array}{cc}
v(\tau)^{2} & s(\tau) v^{\top}(\tau) \\
s(\tau) v^{\top}(\tau) & s(\tau)^{2} I_{3}
\end{array}\right] d \tau \geq \bar{\rho} I_{4}
$$

Now denoting

$$
\begin{aligned}
& \bar{S} \triangleq-s \omega_{\times}+\dot{s} I_{3} \in \mathbb{R}^{3 \times 3} \\
& \bar{v} \triangleq \phi v+\dot{v} \in \mathbb{R}^{3} \\
& H \triangleq\left[g s \hat{R}^{\star \top} e_{2}-g s \hat{R}^{\star \top} e_{1}\right] \in \mathbb{R}^{3 \times 2} \\
& G \triangleq\left[\begin{array}{ll}
H & \bar{v}
\end{array}\right] \in \mathbb{R}^{3 \times 3}
\end{aligned}
$$

one verifies that $C^{\star} A^{\star}+\dot{C}^{\star}=\left[\begin{array}{ll}G & \bar{S}\end{array}\right]$ and, thus,

$$
\begin{aligned}
& M^{\top} M=\left[\begin{array}{cc}
G^{\top} G+|v|^{2} e_{3} e_{3}^{\top} & G^{\top} \bar{S}+s e_{3} v^{\top} \\
\bar{S}^{\top} G+s v e_{3}^{\top} & \overline{S^{\top}} \bar{S}+s^{2} I_{3}
\end{array}\right] \\
& =\left[\begin{array}{cc}
\frac{1}{1+\varepsilon_{1}} G^{\top} G & G^{\top} \bar{S} \\
\bar{S}^{\top} G & \left(1+\varepsilon_{1}\right) \bar{S}^{\top} \bar{S}
\end{array}\right] \\
& +\left[\begin{array}{cc}
\frac{\varepsilon_{1}}{1+\varepsilon_{1}} G^{\top} G+|v|^{2} e_{3} e_{3}^{\top} & s e_{3} v^{\top} \\
s v e_{3}^{\top} & s^{2} I_{3}-\varepsilon_{1} \bar{S}^{\top} \bar{S}
\end{array}\right] \\
& =\left[\begin{array}{cc}
\frac{1}{1+\varepsilon_{1}} G^{\top} G & G^{\top} \bar{S} \\
\bar{S}^{\top} G & \left(1+\varepsilon_{1}\right) \bar{S}^{\top} \bar{S}
\end{array}\right] \\
& +\left[\begin{array}{cc}
\frac{\varepsilon_{1}}{1+\varepsilon_{1}}\left[\begin{array}{cc}
\frac{1}{1+\varepsilon_{2}} H^{\top} H & H^{\top} \bar{v} \\
\bar{v}^{\top} H & \left(1+\varepsilon_{2}\right)|\bar{v}|^{2}
\end{array}\right] & 0_{3 \times 3} \\
0_{3 \times 3} & 0_{3 \times 3}
\end{array}\right]
\end{aligned}
$$

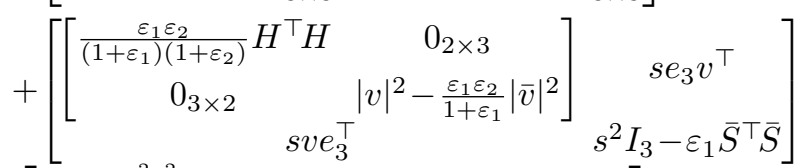

$$
\begin{aligned}
& \geq\left[\begin{array}{cc}
\frac{\varepsilon_{1} \varepsilon_{2} g^{2} s^{2}}{\left(1+\varepsilon_{1}\right)\left(1+\varepsilon_{2}\right)} I_{2} & 0_{2 \times 6} \\
0_{6 \times 2} & {\left[\begin{array}{cc}
|v|^{2}-\frac{\bar{\rho}}{2} & s v^{\top} \\
s v & \left(s^{2}-\frac{\bar{\rho}}{2}\right) I_{3}
\end{array}\right]}
\end{array}\right]
\end{aligned}
$$

with $\varepsilon_{1}=\frac{\bar{\rho} d_{\min }^{2}}{2\left(\omega_{\max }+\phi_{\max }\right)^{2}}, \varepsilon_{2}=\frac{\bar{\rho}\left(1+\varepsilon_{1}\right)}{2 \varepsilon_{1}\left(\phi_{\max } v_{\max }+\dot{v}_{\max }\right)^{2}}$. From (17) and (18), one easily deduces that (15) is satisfied, with $\mu=\min \left(\frac{\varepsilon_{1} \varepsilon_{2} g^{2} s^{2}}{\left(1+\varepsilon_{1}\right)\left(1+\varepsilon_{2}\right)}, \frac{\bar{\rho}}{2}\right)$. This concludes the proof.

Now the persistent excitation condition (16) deserves some comments.
- First, this condition is violated if the vehicle's linear velocity is null for all time (i.e. $|v(t)| \equiv 0$ ). In fact, in this situation it is impossible to recover the depth from monocular vision without any prior knowledge of the observed planar scene.

- If the vehicle does move "persistently" so that $\exists \delta_{v}, \rho_{v}>$ 0 such that

$$
\frac{1}{\delta_{v}} \int_{t}^{t+\delta_{v}}|v(\tau)| d \tau \geq \rho_{v}, \forall t \geq 0
$$

and that Assumption 1 holds, then condition (16) is satisfied for almost all types of motion, excepts some very particular cases. For instance, such is the case where the vehicle moves, with constant linear velocity and constant attitude, in a straight-line path parallel to the observed plane (i.e. $v(t)$ and $d(t)$ remain constant).

\section{Yaw estimation}

For completeness, the third component $\sigma_{R, 3}$ of the innovation term $\sigma_{R}$ is now independently designed for yaw estimation.

Corollary 1 In addition to the innovations $\sigma_{R, 1}, \sigma_{R, 2}$ and $\sigma_{V}$ specified previously, define

$$
\sigma_{R, 3}=-k_{m} e_{3}^{\top}\left(\hat{R} m_{\mathcal{B}} \times m_{\mathcal{I}}\right)
$$

where $k_{m} \in \mathbb{R}$ is either a positive number or $k_{m}=\left(m_{1}^{2}+\right.$ $\left.m_{2}^{2}\right) Q_{m} P_{m}$, with $P_{m} \in \mathbb{R}$ solution to the following CRE:

$$
\dot{P}_{m}=-\left(m_{1}^{2}+m_{2}^{2}\right)^{2} Q_{m} P_{m}^{2}+V_{m}, P_{m}(0)>0
$$

and $Q_{m}, V_{m}$ positive numbers. Then, the equilibrium $(\hat{R}, \hat{v}, \hat{s})=(R, v, s)$ of the proposed Riccati observer is locally exponentially stable.

Proof: As a result of Proposition 1, it suffices to prove the local exponential stability of $\tilde{\lambda}_{3}=0$ at the local zerodynamics of $\tilde{\lambda}_{3}$ by setting $\tilde{\lambda}_{1} \equiv \tilde{\lambda}_{2} \equiv 0$ and $\tilde{v} \equiv 0$. One verifies that the zero-dynamics of $\tilde{\lambda}_{3}$ are locally given by

$$
\dot{\tilde{\lambda}}_{3}=\sigma_{R, 3}
$$

while the "conditioned" magnetometer measurement $e_{3}^{\top}\left(\hat{R} m_{\mathcal{B}} \times m_{\mathcal{I}}\right)$ in first order approximations and with $\tilde{\lambda}_{1} \equiv \tilde{\lambda}_{2} \equiv 0$ is approximately given by

$$
e_{3}^{\top}\left(\hat{R} m_{\mathcal{B}} \times m_{\mathcal{I}}\right)=\left(m_{1}^{2}+m_{2}^{2}\right) \tilde{\lambda}_{3}
$$

so that

$$
\dot{\tilde{\lambda}}_{3}=-k_{m}\left(m_{1}^{2}+m_{2}^{2}\right) \tilde{\lambda}_{3}
$$

From here the proof straightforwardly follows.

\section{Practical implementation aspects}

\section{A. Unit quaternion equivalence}

Although the attitude estimate is designed on $S O(3)$, it can be directly lifted to an equivalent algorithm on the unit quaternion group (see, e.g., [14]). Let $\hat{q}$ denote the unit quaternion associated with $\hat{R}$. Then, the proposed observer (10) can be rewritten as

$$
\left\{\begin{array}{l}
\dot{\hat{q}}=\frac{1}{2}\left(\Gamma_{1}(\omega)-\Gamma_{2}\left(\sigma_{R}\right)\right) \hat{q} \\
\hat{\hat{v}}=-[\omega]_{\times} \hat{v}+a_{\mathcal{B}}+g \hat{R}^{\top} e_{3}-\sigma_{v} \\
\dot{\hat{s}}=\phi \hat{s}-\sigma_{s}
\end{array}\right.
$$

where the mappings $\Gamma_{1}, \Gamma_{2}: \mathbb{R}^{3} \rightarrow \mathbb{R}^{4 \times 4}$ are defined as 


$$
\Gamma_{1}(x)=\left[\begin{array}{cc}
0 & -x^{\top} \\
x & -[x]_{\times}
\end{array}\right], \Gamma_{2}(x)=\left[\begin{array}{cc}
0 & -x^{\top} \\
x & {[x]_{\times}}
\end{array}\right], \forall x \in \mathbb{R}^{3}
$$

and the term $\hat{R}$ is calculated from $\hat{q}$ using the Rodrigues formula.

\section{B. Hybrid discrete-continuous version}

In practice, the IMU measurements can be obtained at a very high frequency while the continuous homography is often estimated at a much lower frequency. This fact should be carefully taken into account in the implementation process. Inspired by existing hybrid continuous-discrete Kalman or extended Kalman filters, we propose thereafter a hybrid continuous-discrete version of the proposed observer, where for the sake of simplicity the gain $k_{m}$ involved in the expression (20) of the innovation component $\sigma_{R, 3}$ is a positive number.

Let $\left\{t_{k}\right\}$ denote the suite of time-instants that the continuous homography estimates are obtained. Then, the prediction and correction steps of the proposed observer are described below.

- Prediction step: At each step $k$, integrate during $t \in$ $\left[t_{k-1}, t_{k}\right]$ the following equations

$$
\left\{\begin{array}{l}
\dot{\hat{q}}=\frac{1}{2}\left(\Gamma_{1}(\omega)-\Gamma_{2}\left(\sigma_{R, 3} e_{3}\right)\right) \hat{q} \\
\hat{\hat{v}}=-[\omega]_{\times} \hat{v}+a_{\mathcal{B}}+g \hat{R}^{\top} e_{3} \\
\dot{\hat{s}}=\phi \hat{s} \\
\dot{P}=A P+P A^{\top}+V
\end{array}\right.
$$

with $\hat{q}\left(t_{k-1}\right)=\hat{q}_{k-1 \mid k-1}, \hat{v}\left(t_{k-1}\right)=\hat{v}_{k-1 \mid k-1}, \hat{s}\left(t_{k-1}\right)=$ $\hat{s}_{k-1 \mid k-1}, P\left(t_{k-1}\right)=P_{k-1 \mid k-1}$ to obtain

$$
\left\{\begin{array}{l}
\hat{q}_{k \mid k-1}=\hat{q}\left(t_{k}\right) \\
\hat{v}_{k \mid k-1}=\hat{v}\left(t_{k}\right) \\
\hat{s}_{k \mid k-1}=\hat{s}\left(t_{k}\right) \\
P_{k \mid k-1}=P\left(t_{k}\right)
\end{array}\right.
$$

- Correction step: First, compute the innovation terms as

$$
\left\{\begin{aligned}
K_{k} & =P_{k \mid k-1} C_{k}^{\top}\left(C_{k} P_{k \mid k-1} C_{k}^{\top}+Q^{-1}\right)^{-1} \\
u_{k} & =\left[\begin{array}{c}
\sigma_{R k, 1} \\
\sigma_{R k, 2} \\
\sigma_{s k} \\
\sigma_{v k}
\end{array}\right]=-K_{k} y_{k}
\end{aligned}\right.
$$

with $C_{k}=\left[\begin{array}{llll}0_{3 \times 1} & 0_{3 \times 1} & \hat{v}_{k \mid k-1} & \hat{s}_{k \mid k-1} I_{3}\end{array}\right], y_{k}=\left(\frac{v}{d}\right)_{k}-$ $\hat{v}_{k \mid k-1} \hat{s}_{k \mid k-1}$. Then, update the state estimates and the Riccati matrix as

$$
\begin{aligned}
& \qquad\left\{\begin{array}{l}
\hat{q}_{k \mid k}=\exp \left(-\frac{1}{2} \Gamma_{2}\left(\sigma_{R k, 1} e_{1}+\sigma_{R k, 2} e_{2}\right)\right) \hat{q}_{k \mid k-1} \\
\hat{v}_{k \mid k}=\hat{v}_{k \mid k-1}-\sigma_{v k} \\
\hat{s}_{k \mid k}=\hat{s}_{k \mid k-1}-\sigma_{s k} \\
P_{k \mid k}=\left(I_{6}-K_{k} C_{k}\right) P_{k \mid k-1}
\end{array}\right. \\
& \text { with } \operatorname{sinc}(x)=\sin (x) / x, \forall x \in \mathbb{R} .
\end{aligned}
$$

\section{Practical solutions for the boundedness of $P$}

As mentioned in Section III-B the Riccati matrix $P$ is well conditioned provided that the pair $\left(A^{\star}, C^{\star}\right)$ given in (14) is uniformly observable (i.e. the persistent excitation condition (16) is satisfied). However, when this uniform observability condition is violated (as discussed in the end of Section IIIB) $P$ may grow arbitrarily large or even explode. Some "practical" solutions to that issue are proposed next. For instance, when the measured quantity $\frac{v}{d}$ is not null, it is likely that condition (16) is satisfied. Therefore, when the norm of $\frac{v}{d}$ is smaller than some small threshold, one can simply inactivate the correction step and also the integration of $P$ within the prediction step. Another solution consists in saturating $P$ after every correction step so that its Frobenius norm remains always smaller than a given threshold. The latter should be chosen large enough so that the saturation of $P$ will not occur when the system is uniformly observable (i.e. sufficiently excited).

\section{EXPERIMENTAL EVALUATION}

\section{A. Experimental setup}

For experimental validations, we make use of a VisualInertial (VI) sensor developed by the Autonomous Systems Lab (ETH Zurich) and the company Skybotix [20]. Among the two cameras and two IMUs of the VI-sensor, only one camera and one IMU (composed of a 3-axis gyrometer and a 3-axis accelerometer) are used to validate the proposed algorithm. The main reason for using the VI-sensor in this experimental setup is the possibility of obtaining perfectly time-synchronized images and IMU readings $(20 \mathrm{~Hz}$ for the camera and $200 \mathrm{~Hz}$ for the IMU). On the other hand, the OptiTrack motion capture system available at I3S is used to obtain the ground truth data for comparison purposes. This highly accurate OptiTrack system provides the full pose of the Camera-IMU system at $120 \mathrm{~Hz}$. As a matter of fact, the quantities such as $\frac{v}{d}$ and $\phi$ can be emulated from the ground-truth pose measurements. For instance, using the position measurements a high-gain observer is applied for the estimation of the linear velocity expressed in the inertial frame that is then converted to the linear velocity expressed in the body-fixed frame using the ground-truth attitude. Then, by considering the situation where the UAV carries a downward-looking camera to observe a planar horizontal ground, it is not difficult to construct the "measurements" of $\frac{v}{d}$ and $\phi($ at $20 H z)$.

The hybrid discrete-continuous version of the proposed observer has been implemented in $\mathrm{C}++$, combined with OpenCV for image processing, on an Intel Core i7-6400 CPU running at $3.40 \mathrm{Ghz}$. The transmission of data from the camera to the PC is carried out through a high speed ethernet cable. The PC has a Linux based operating system and is responsible for two major software tasks:

- Interface with the camera hardware and acquisition of images and IMU data from the VI-sensor.

- Estimation of the continuous homography based on two consecutive images which is then decomposed to obtain the measurements of $\frac{v}{d}$ and $\phi$ in real-time.

Due to real-time constraint for the continuous homography estimation, feature detection and descriptor extraction in images are carried out using the FAST Feature Detector ${ }^{2}$ and ORB Descriptor Extractor algorithms already implemented in the OpenCV library. Since the quality of the continuous

\footnotetext{
${ }^{2}$ Although FAST is less robust than other algorithms such as SIFT or SURF, it is much faster and more suitable for real-time implementation.
} 
homography estimation depends heavily on the capability of rejecting outliers of point matchings, we have implemented an M-estimator-like observer for the estimation of the homography between every two consecutive images, which is then used to compute the continuous homography via a logarithm conversion. This M-estimator-like homography observer is a modified version of the homography observer proposed in [13] but is not presented here due to space limitation. However, the reader can appreciate its performance and robustness via the following video link:

https://youtu.be/x75RpjoJ9HM

Although the combined implementation of image processing and estimation algorithm runs at about $50 \mathrm{~Hz}$, the continuous homography estimate is only obtained at every $50 \mathrm{~ms}(20 \mathrm{~Hz})$ due to the lower acquisition frequency of the VI-sensor camera.

\section{B. Experimental results}

The reported experiment has been performed online with the VI-sensor camera looking downward to observe a well textured planar horizontal ground. A demo video is provided as a supplemental material and is also available at https://youtu.be/R09oTjr4s40
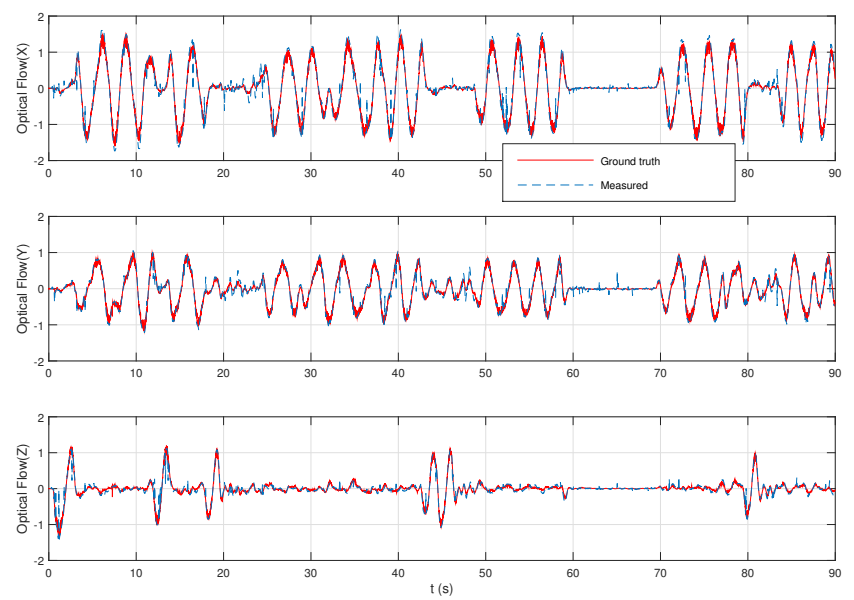

Fig. 1. (Experiment) Optical flow components measured from images (blue curves) and derived from ground truth pose (red curves) versus time $(s)$

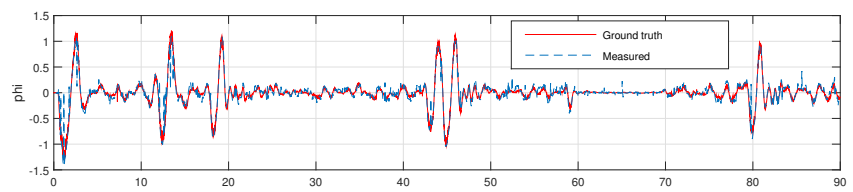

Fig. 2. (Experiment) $\phi=-\frac{\dot{d}}{d}$ measured from images (blue curve) and derived from ground truth pose (red curve) versus time $(s)$

The parameters involved in the proposed observer are chosen as follows: $Q=\operatorname{diag}\{8,8,24\}, V=$ $\operatorname{diag}\left\{0.02^{2} I_{2}, 0.1^{2}, 0.2^{2} I_{3}\right\}$ and $P(0)=1.7 I_{6}$. The initial estimates are given by $\hat{q}(0)=[1,0,0,0], \hat{v}(0)=$ $[0,0,0](\mathrm{m} / \mathrm{s}), \hat{s}(0)=4\left(\mathrm{~m}^{-1}\right)$.

Figs. 1 and 2 show a good quality of the optical flow $\frac{v}{d}$ as well as $\phi\left(=-\frac{\dot{d}}{d}\right)$ obtained from the decomposition of the continuous homography estimate when compared to the corresponding ground-truth data.
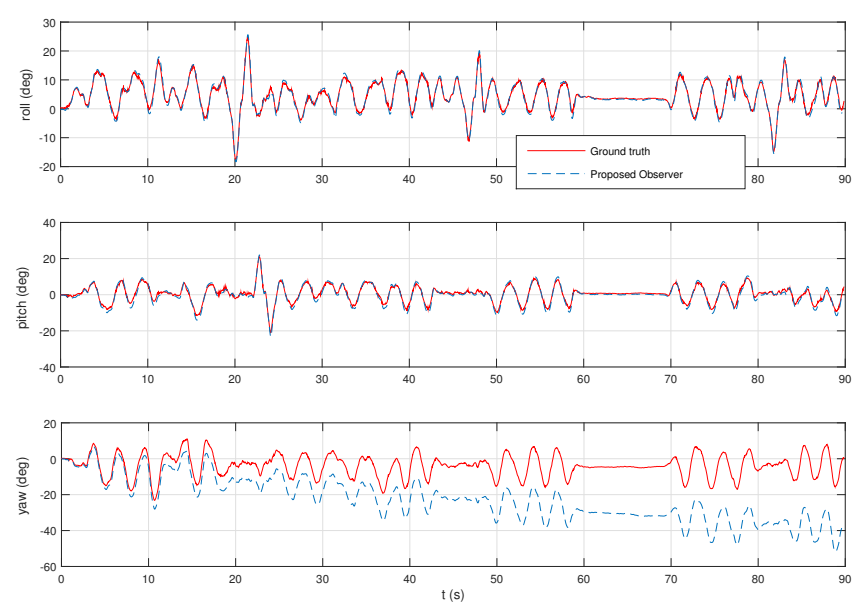

Fig. 3. (Experiment) Estimated and ground-truth attitudes represented by roll, pitch and yaw Euler angles $(\mathrm{deg})$ versus time $(s)$
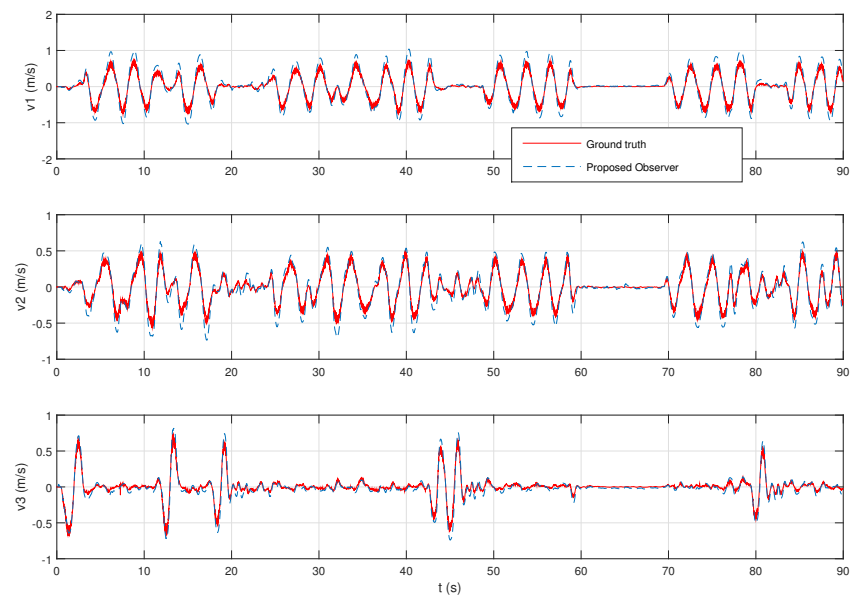

Fig. 4. (Experiment) Estimated and ground-truth linear velocity components in body-fixed frame $(\mathrm{m} / \mathrm{s})$ versus time $(\mathrm{s})$

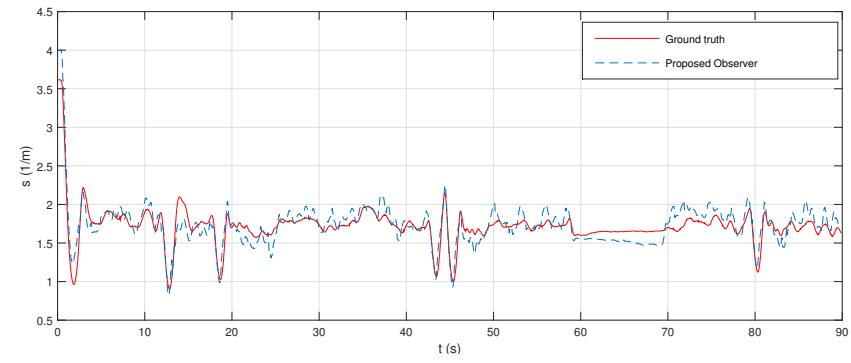

Fig. 5. (Experiment) Estimated and ground-truth depth inverse $\left(m^{-1}\right)$ versus time $(s)$

The time evolutions of the estimated and ground-truth attitudes, body-fixed linear velocities and depths are depicted in Figs. 3-5. During the first 60 seconds, it can be observed that the depth estimate as well as both the estimated gravity direction (i.e. roll and pitch Euler angles) and the estimated linear velocity converge near to the corresponding groundtruth values since the condition of persistent excitation is preserved. In contrast, during the period of 60 to 69 seconds when the camera is kept still thus violating the condition of persistent excitation, it can be seen that the depth estimate slightly drifts away from the ground-truth depth, whereas both the estimated gravity direction and linear 
velocity always remain close to the corresponding groundtruth values. Once the condition of persistent excitation is revoked again by moving the camera from 69 to 89 seconds, the depth estimate follows closely again the ground-truth value along with the gravity direction and linear velocity estimates. From Fig. 3 it can also be observed that the yaw angle estimate drifts away from the ground-truth value. This is normal since it is simply an integration of the gyros (i.e. magnetometer measurements are not used for yaw estimation correction in this experiment). In conclusion, the reported experiment shows that whereas the (practical) convergence of the gravity direction and linear velocity estimates is always achieved, the convergence of the depth estimate is additionally obtained only when the condition of persistent excitation is guaranteed.

\section{CONCLUSIONS}

In this paper we discussed the observability issue of the attitude, the linear velocity and the depth of a Camera with respect to a planar target from optical flow measurements along with IMU data. The proposed nonlinear deterministic observer is effective when the camera's linear velocity satisfies a condition of persistent excitation. Experimental results have been provided as a complement to the theoretical approach to show the performance of the observer and the practical convergence of the observer errors. The proposed solution provides a natural plug-and-play capability for applications in which the aerial robot interacts actively with its surrounding environment and a human operator. It is well suited to haptic feedback control of a UAV (stabilization of reference linear velocity of the UAV) while the optical flow can be used as a cue for haptic force feedback [9], [21]. This is part of our future work plans.

\section{ACKNOWLEDGMENT}

This work was supported by the FUI GreenExplorer project and the EQUIPEX Robotex project.

\section{REFERENCES}

[1] G. Allibert, R. Mahony, and M. Bangura. Velocity aided attitude estimation for aerial robotic vehicles using latent rotation scaling. In IEEE Int. Conf. on Robotics and Automation, pages 1538-1543, 2016.

[2] S. Bonnabel, P. Martin, and P. Rouchon. Symmetry-preserving observers. IEEE Trans. on Autom. Control, 53(11):2514-2526, 2008.

[3] D. Brescianini, M. Hehn, and R. D'Andrea. Quadrocopter pole acrobatics. In IEEE/RSJ International Conference on Intelligent Robots and Systems, pages 3472-3479, 2013.

[4] V. Grabe, H.H. Bülthoff, and P.R. Giordano. On-board velocity estimation and closed-loop control of a quadrotor UAV based on optical flow. In IEEE International Conference on Robotics and Automation (ICRA), pages 491-497, 2012.

[5] V. Grabe, H.H. Bülthoff, D. Scaramuzza, and P.R. Giordano. Nonlinear ego-motion estimation from optical flow for online control of a quadrotor uav. The International Journal of Robotics Research, 34(8):1114-1135, 2015.

[6] H.F. Grip, T.I. Fossen, T.A. Johansen, and A. Saberi. A nonlinear observer for integration of GNSS and IMU measurements with gyro bias estimation. In American Cont. Conf., pages 4607-4612, 2012.

[7] B.J. Guerreiro, C. Silvestre, R. Cunha, and D. Cabecinhas. LiDARBased control of autonomous rotorcraft for the inspection of pierlike structures. IEEE Transactions on Control Systems Technology, 2017.

[8] T. Hamel and C. Samson. Riccati observers for the non-stationary PnP problem. IEEE Trans. on Automatic Control (in press), 2017.
[9] X. Hou and R. Mahony. Dynamic kinesthetic boundary for haptic teleoperation of aerial robotic vehicles. In IEEE/RSJ International Conference on Intelligent Robots and Systems (IROS), pages 45494950, 2013.

[10] M.-D. Hua. Attitude estimation for accelerated vehicles using GPS/INS measurements. Control Eng. Pract., 18(7):723-732, 2010.

[11] M.-D. Hua, T. Hamel, and C. Samson. Riccati observers for velocityaided attitude estimation of accelerated vehicles using coupled velocity measurements. In To appear in IEEE Conference on Decision and Control (CDC), 2017. Available at arXiv preprint arXiv:1703.07252.

[12] M.-D. Hua, P. Martin, and T. Hamel. Stability analysis of velocityaided attitude observers for accelerated vehicles. Automatica, 63:1115, 2016.

[13] M.-D. Hua, J. Trumpf, T. Hamel, R. Mahony, and P. Morin. Featurebased recursive observer design for homography estimation. arXiv preprint arXiv:1606.03021, 2016.

[14] M.-D. Hua, M. Zamani, J. Trumpf, R. Mahony, and T. Hamel. Observer design on the Special Euclidean group SE(3). In IEEE Conference on Decision and Control, pages 8169-8175, 2011.

[15] A. Khosravian, J. Trumpf, R. Mahony, and T. Hamel. State estimation for invariant systems on lie groups with delayed output measurements. Automatica, 68:254-265, 2016.

[16] R.C. Leishman, J.C. Macdonald, R.W. Beard, and T.W. McLain Quadrotors and accelerometers: State estimation with an improved dynamic model. IEEE Control Systems, 34(1):28-41, 2014.

[17] Y. Ma, S. Soatto, J. Kosecka, and S.S. Shankar. An invitation to 3-D vision: from images to geometric models, volume 26. Springer Science \& Business Media, 2012.

[18] P. Martin and E. Salaün. An invariant observer for Earth-VelocityAided attitude heading reference systems. In IFAC World Congr., pages 9857-9864, 2008.

[19] P. Martin and I. Sarras. A semi-global model-based state observer for the quadrotor using only inertial measurements. In IEEE Conference on Decision and Control (CDC), pages 7123-7128, 2016.

[20] J. Nikolic, J. Rehder, M. Burri, P. Gohl, S. Leutenegger, P.T. Furgale, and R. Siegwart. A synchronized visual-inertial sensor system with FPGA pre-processing for accurate real-time SLAM. In IEEE International Conference on Robotics and Automation (ICRA), pages 431-437, 2014.

[21] S. Omari, M.-D. Hua, G. Ducard, and T. Hamel. Bilateral Haptic Teleoperation of VTOL UAVs. In IEEE International Conference on Robotics and Automation (ICRA), pages 2393-2399, 2013.

[22] A. Roberts and A. Tayebi. On the attitude estimation of accelerating rigid-bodies using GPS and IMU measurements. In IEEE Conf. on Dec. and Cont., pages 8088-8093, 2011.

[23] G.G. Scandaroli. Visuo-inertial data fusion for pose estimation and self-calibration. PhD thesis, Université Nice Sophia Antipolis, 2013.

[24] D. Scaramuzza, M.C. Achtelik, L. Doitsidis, F. Friedrich, et al. Visioncontrolled micro flying robots: from system design to autonomous navigation and mapping in GPS-denied environments. IEEE Robotics \& Automation Magazine, 21(3):26-40, 2014.

[25] T. Tang, K. Sreenath, and V. Kumar. Aggressive maneuvering of a quadrotor with a cable-suspended payload. In Robotics: Science and Systems, Workshop on Women in Robotics, 2014.

[26] J. Thomas, M. Pope, G. Loianno, E.L. Elliot, M.A. Estrada, H. Jiang, M.R. Cutkosky, and V. Kumar. Aggressive flight with quadrotors for perching on inclined surfaces. Journal of Mechanisms and Robotics, 8(5):051007-051007-10, 2016.

[27] S. Weiss, R. Brockers, and L. Matthies. 4DoF drift free navigation using inertial cues and optical flow. In IEEE/RSJ International Conference on Intelligent Robots and Systems (IROS), pages 41804186, 2013. 